\section{The impact of early discontinuation/dose modification of venetoclax on outcomes in patients with relapsed/refractory chronic lymphocytic leukemia: post-hoc analyses from the phase III MURANO study}

Haematologica 2022

Volume 107(1):134-142

\section{Correspondence:}

ANTHONY R. MATO

matoa@mskcc.org

Received: July 10, 2020.

Accepted: November 27, 2020.

Pre-published: December 17, 2020.

https://doi.org/10.3324/haematol.2020.266486

(C)2022 Ferrata Storti Foundation

Material published in Haematologica is covered by copyright. All rights are reserved to the Ferrata Storti Foundation. Use of published material is allowed under the following terms and conditions:

https://creativecommons.org/licenses/by-nc/4.0/legalcode. Copies of published material are allowed for personal or internal use. Sharing published material for non-commercial pur-

https://creativecommons.org/licenses/by-nc/4.0/legalcode, sect. 3. Reproducing and sharing published material for commercial purposes is not allowed without permission in writing from the publisher. poses is subject to the following conditions:

\section{Anthony R. Mato, ${ }^{1}$ Jeff P. Sharman, ${ }^{2}$ Juliana M.L. Biondo, ${ }^{3}$ Mei Wu, ${ }^{3}$ Yong Mun, ${ }^{3}$ Su Y. Kim, ${ }^{4}$ Kathryn Humphrey, ${ }^{5}$ Michelle Boyer, ${ }^{5}$ Qian $\mathrm{Zhu}^{3}$ and John F. Seymour ${ }^{6}$}

${ }^{1}$ Memorial Sloan Kettering Cancer Center, New York, NY, USA; ${ }^{2}$ Willamette Valley Cancer Institute and Research Center, Eugene, OR, USA/US Oncology Research, US Oncology Network, The Woodlands, TX, USA; ${ }^{3}$ Genentech, Inc., South San Francisco, CA, USA; ${ }^{4}$ AbbVie, North Chicago, IL, USA; ${ }^{5}$ Roche Products Limited, Welwyn Garden City, UK and ${ }^{6}$ Peter MacCallum Cancer Centre, Royal Melbourne Hospital, The University of Melbourne, Melbourne, Victoria, Australia
Tixed-duration venetoclax plus rituximab (VenR) has a manageable Isafety profile and improves survival in patients with relapsed/refractory (R/R) chronic lymphocytic leukemia (CLL). We present data from the phase III MURANO study on the impact of venetoclax modification or premature discontinuation on outcomes in patients with R/R CLL. Timedependent Cox proportional hazards regression models, stratified by $17 p$ deletion and risk status, evaluated the impact of venetoclax discontinuation/modification on investigator-assessed progression-free survival (PFS) and overall survival (OS). Analyses were performed retrospectively (without type-1 error control) in intention-to-treat patients from the VenR arm of MURANO. Overall, 140 of 194 (72\%) patients in the VenR arm completed 2 years of therapy; 54 of 194 (28\%) patients prematurely discontinued treatment. Inferior PFS was observed in patients prematurely discontinuing venetoclax for any reason (disease progression excluded; $P<0.0001)$ and specifically in patients discontinuing due to adverse event (AE) $(P<0.0001)$, versus those who did not discontinue early. Risk of a $\mathrm{PFS} / \mathrm{OS}$ event was significantly reduced by each extra month (exposure cycle) of venetoclax therapy ( $P=0.0263$ for PFS; $P<0.0001$ for OS). Treatment interruption for AE occurred in 134 of 194 (69\%) patients, most commonly due to neutropenia (84 of $194 ; 43 \%$ ), per protocol requirements. Treatment interruption had no impact on PFS or OS, regardless of duration. Dose reductions were required by 45 of 194 (23\%) patients, but had no significant impact on outcomes. In MURANO, premature discontinuation was associated with suboptimal outcomes; venetoclax treatment modification was not. These data highlight the importance of effective toxicity control to realize the full benefit of venetoclax treatment (clinicaltrials gov. Identifier: NCT02005471).

\section{Introduction}

Chronic lymphocytic leukemia (CLL), the most common adult leukemia in Western countries, accounts for approximately one-third of new adult leukemia diagnoses in the United States. ${ }^{1,2}$ Although no curative treatment for CLL exists (apart from allogeneic stem cell transplantation, which is unsuitable for most patients), ${ }^{3}$ chemoimmunotherapy achieves disease control and prolongs survival, and has historically been the standard treatment. More recently, novel targeted therapies have improved outcomes over chemoimmunotherapy in first-line (1L) and relapsed/refractory (R/R) CLL. ${ }^{4}$ These comprise selective kinase inhibitors targeting the B-cell receptor and its downstream proteins (including the Bruton tyro- 
sine kinase [BTK] inhibitors ibrutinib and acalabrutinib, and the phosphatidylinositol 3-kinase [PI3K] inhibitors idelalisib and duvelisib) and the potent B-cell lymphoma2 (BCL-2) inhibitor venetoclax. ${ }^{5}$

Mechanisms of action differ between targeted therapies. BTK inhibitors reduce CLL cell chemotaxis toward the chemokines CXCL12 and CXCL13 and block B-cell receptor signaling. ${ }^{6}$ This drives apoptosis and/or disrupts cell migration and adherence to protective tumor microenvironments. PI3K inhibitors impede one or more of the PI3K enzymes, part of the PI3K/AKT/mTOR pathway that regulates many cellular functions (e.g., growth control, metabolism and translation initiation), resulting in tumor suppression. Venetoclax is a BH3-mimetic, which blocks the anti-apoptotic BCL-2 protein, leading to programmed cell death of CLL cells. ${ }^{8}$

Ibrutinib, idelalisib, duvelisib and acalabrutinib are used until progression or toxicity, while venetoclax provides a chemotherapy-free treatment option that allows for a finite duration of therapy. ${ }^{9}$ Venetoclax has a fixed treatment duration of 1 year when given concomitantly with obinutuzumab for $1 \mathrm{~L}$ CLL, 2 years when given in combination with rituximab for R/R CLL, and until disease progression (PD) or toxicity as a monotherapy for R/R CLL. ${ }^{10-13}$

Although targeted therapies have manageable safety profiles and in some instances improve life expectancy for patients with CLL, both premature discontinuations (before PD) and modifications to therapy are reported in a proportion of cases. To date, information on whether premature discontinuations and/or treatment modifications have an impact on survival outcomes in patients with CLL (in both real-world studies and clinical trials) has not been summarized collectively. However, numerous publications address this question, with significant literature available on the impact of disruption of ibrutinib on survival (Online Supplemental Table S1). ${ }^{14-28}$ In brief, the literature suggests that ibrutinib discontinuation is associated with poor survival outcomes, ${ }^{16,19,21}$ while the impact of interruption on outcomes seems contradictory (with some indicating an effect on survival and others suggesting a lack of difference in outcomes). ${ }^{14,15,18-20,22,23}$ Limited data are available for venetoclax; and given the different mechanism of action, more rapid kinetics of response and deeper remissions with venetoclax (especially when used with anti-CD20 antibodies), extrapolation of ibrutinib data is unlikely to be valid for venetoclax.

The MURANO phase III, international, randomized, open-label study evaluated the efficacy of six cycles of venetoclax in combination with rituximab (VenR) then venetoclax once daily for 2 years in total compared with six cycles of bendamustine plus rituximab (BR) in patients with R/R CLL. ${ }^{29}$ VenR was superior to BR; after a median follow-up period of 23.8 months, investigator-assessed progression-free survival (PFS) was significantly longer in the VenR group (32 events in 194 patients) than in the BR group (114 events in 195 patients). ${ }^{29}$ Additionally, PFS benefit of VenR over BR was sustained (hazard ratio [HR], 0.19 [95\% Confidence Interval [CI]: 0.14-0.25]; $P<0.0001)$, with 4 -year PFS estimates of $57.3 \%$ (95\% CI: 49.4-65.3) versus $4.6 \%$ (95\% CI: $0.1-9.2$ ), respectively. ${ }^{30}$ While the minimum duration of venetoclax necessary to achieve therapeutic benefit remains unclear, the available data suggest that no substantial further deepening of responses occurs beyond 24 months of therapy. ${ }^{31}$
This manuscript reports novel venetoclax data from post-hoc analyses of MURANO to assess the impact of discontinuation/modification on outcomes and provides the first review of the current published literature (for all oral targeted agents approved for use in CLL as of August 2019: ibrutinib, idelalisib and duvelisib; see the Online Supplemental Appendix), to contextualize these data.

\section{Methods}

\section{MURANO study methodology}

Analyses were performed on data from the VenR cohort of patients in the MURANO study (NCT02005471), which has been described previously. ${ }^{29}$

MURANO enrolled patients aged $\geq 18$ years, who had received 1-3 prior lines of therapy (including at least one chemotherapycontaining regimen), had an Eastern Cooperative Oncology Group performance status of 0 or 1 , and had adequate bone marrow, renal, and hepatic function. Patients received venetoclax over a 5week dose ramp-up period (to reach the $400 \mathrm{mg}$ daily target dose) followed by six cycles of rituximab (single infusion on day 1 of each 28-day cycle: $375 \mathrm{mg} / \mathrm{m}^{2}$ on day 1 , cycle 1 and $500 \mathrm{mg} / \mathrm{m}^{2}$ for day 1, cycles 2-6) with ongoing daily dosing of venetoclax. Patients continued daily venetoclax therapy for a maximum of 2 years (from day 1, cycle 1). All relevant ethical committees approved the MURANO study.

\section{Data and statistical analyses}

Efficacy analyses were based on the intention-to-treat population (all patients who underwent randomization). After trial drug initiation, all adverse events (AE), including those resulting in discontinuation or treatment modification (including treatment interruption and dose reduction), were reported up to 28 days after the last dose of trial drug (maximum of 2 years) or up to 90 days after the last dose of rituximab, whichever was longer. Discontinuation and modification events were recorded (see the Online Supplementary Appendix). Primary endpoints of the post-hoc analyses included investigator-assessed PFS and overall survival (OS). PFS was defined as time from randomization to first occurrence of $\mathrm{PD}$, relapse or death.

In order to determine the effect of treatment discontinuations and modifications on clinical outcomes, eight time-dependent Cox proportional hazards regression models stratified by chromosome 17p deletion ( $\operatorname{del}(17 \mathrm{p})$ ) and disease risk status (high or low risk; see the Online Supplementary Appendix) were used to evaluate investigator-assessed PFS and OS (Table 1). Time-fixed baseline covariates and time-dependent serious $\mathrm{AE}(\mathrm{SAE})$ indicators were considered in each model. Time-dependent analyses were performed to account for varying time when treatment discontinuation/modification occurred, helping to assure that longer PFS and OS were not falsely attributed to patients who simply had treatment discontinuation/modification occurring at the later time (i.e., immortal-time bias).

The covariate selection process for the final multivariate model consisted of a bivariate or trivariate time-dependent Cox proportional-hazards regression model stratified by del(17p) and risk status with independent time-dependent variable (variables) and the given time-fixed covariate. Covariates were included in the final multivariate model if the $P$-value of the given time-fixed covariate was $<0.1$. The final multivariate time-dependent Cox proportional-hazards regression model was then stratified by del(17p) and risk status and included time-dependent covariates in addition to the significant time-fixed covariates $(P<0.1$; Table $1)$. The complete-case method was used in the analyses, which 
included patients without missing values for all covariates used in the final multivariate model. All analyses performed were posthoc and are therefore considered hypothesis-generating, with no statistical adjustment for type-I error. A $P$-value of $<0.05$ was considered significant except for in the covariate selection process described above. Data cut-off for this analysis was May 8, 2019

\section{Results}

In total, 194 patients were included in the ITT population for the VenR arm of the MURANO study, of whom 140 completed 2 years of venetoclax therapy. Patient demographics and baseline characteristics have been reported previously. ${ }^{29}$ Half of the patients were aged $<65$ years $(50.0 \%)$, most patients were male $(70.1 \%)$, white $(93.3 \%)$ and had Rai stage $0-$ II at enrollment (71.6\%). In total, 46 of 173 patients $(26.6 \%)$ who were assessed for $\operatorname{del}(17 p)$ status had this deletion, 48 of 192 patients $(25.0 \%)$ who were tested for TP53 mutation status had TP53 mutations, and 123 of 180 patients $(68.3 \%)$ who were tested for immunoglobulin heavy chain variable region (IGVH) mutational status had unmutated IGVH.

\section{Treatment discontinuation}

Early discontinuation of venetoclax ( $<2$ years) was reported in $27.8 \%$ of patients (54 of 194). The two most common reasons for discontinuation was AE (29 of 54, $53.7 \%$ ), followed by PD (12 of $54,22.2 \%$ ). Two of the remaining 13 patients died, and the other 11 discontinued due to withdrawal by patient $(n=5)$, physician decision $(n=3)$, other reasons $(n=2)$ and non-compliance $(n=1)$. In total, 22 of $28(79 \%)$ deaths reported for the MURANO study were in patients who discontinued venetoclax prematurely. Of those, 13 of $22(59.1 \%)$ deaths were in patients who discontinued venetoclax due to $\mathrm{AE}$ (two were related to venetoclax), while four of $22(18.2 \%)$ deaths were in patients who discontinued due to PD (Table 2). Two of the remaining five of $22(22.7 \%)$ deaths were in patients who discontinued due to death, and three were in patients who discontinued due to withdrawal by patient $(n=1)$, physician decision $(n=1$; related to venetoclax) and other reasons ( $\mathrm{n}=1$; Table 2 ).

Table 1. MURANO: stratified time-dependent Cox proportional-hazards regression models used to assess clinical outcomes.

$\begin{array}{ll}\text { Tilme-dependent } & \text { Clinical } \\ \text { indicators } & \text { outeome }\end{array}$

Model 1 Discontinuation due to any reason excluding patients who discontinued venetoclax due to PD

Model 2 Discontinuation due to $\mathrm{AE}$

Discontinuation due to PD/other

Discontinuation due to venetoclax-related AEs

Model 3 Venetoclax interruption of $\geq 8$ consecutive days

PFS

Model 4 Venetoclax interruption of $\geq 14$ consecutive days

Model 5 Venetoclax interruption of $\geq 21$ consecutive days

Model 6 Venetoclax interruption of $\geq 1$ consecutive days

Model 7 Cumulative treatment (cycle) exposure excluding patients who discontinued venetoclax due to PD

Model 8 Time-dependent venetoclax dose reduction indicator (Yes/No)

PFS

\section{Covariates included in final multivariate model}

TP53 mutation status, number of prior regimens, refractoriness to most recent prior therapy, hepatic impairment status and SAE time-dependent indicator

PFS Age, TP53 mutation status, IGVH mutation status, number of prior regimens, refractoriness to most recent prior therapy, hepatic impairment status and SAE time-dependent indicator

TP53 mutation status, IGVH mutation status, refractoriness to most recent prior therapy and SAE time-dependent indicator

TP53 mutation status, IGVH mutation status, bulky disease, refractoriness to most recent prior therapy and SAE time-dependent indicator

TP53 mutation status, number of prior regimens and refractoriness to most recent prior therapy

TP53 mutation status, IGVH mutation status, bulky disease, refractoriness to most recent prior therapy and SAE time-dependent indicator

TP53 mutation status, number of prior regimen and refractoriness to most recent prior therapy

TP53 mutation status, IGVH mutation status, bulky disease, refractoriness to most recent prior therapy and SAE time-dependent indicator

TP53 mutation status, number of prior regimen and refractoriness to most recent prior therapy

TP53 mutation status, IGVH mutation status, bulky disease, refractoriness to most recent prior therapy and SAE time-dependent indicator

TP53 mutation status, number of prior regimen and refractoriness to most recent prior therapy

PFS TP53 mutation status, IGVH mutation status and SAE time-dependent indicator

OS Chromosome 1lq deletion, TP53 mutation status, Rai stage, number of prior regimen and refractoriness to most recent prior therapy

PFS TP53 mutation status, IGVH mutation status, bulky disease, refractoriness to most recent prior therapy and SAE time-dependent indicator

OS TP53 mutation status, number of prior regimens and refractoriness to most recent prior therapy

AE: adverse event; IGVH: immunoglobulin heavy chain gene; OS: overall survival; PD: progressive disease; PFS: progression-free survival; SAE: serious adverse event. 
Median PFS and median OS for early discontinuation due to any reason except PD was 24.3 months (95\% CI: 20.8-31.9) and not reached (NR; 95\% CI: 24.3-NR), respectively, compared with 52.3 (95\% CI: 47.9-NR) and NR for all patients in the VenR arm and NR $(95 \% \mathrm{CI}$ : 52.3-NR) and NR in patients who completed venetoclax treatment. Discontinuing treatment early (for any reason except PD) was significantly associated with shorter PFS ( $n=181$; HR 5.98, 95\% CI: 3.31-10.82; $P<0.0001)$. A similar adverse impact on PFS was observed for patients who discontinued treatment due to an AE ( $\mathrm{n}=174$; HR 5.82, 95\% CI: 2.93-11.57; $P<0.0001$ ); this inferior survival was consistent with treatment discontinuation due to venetoclax-related AE $(n=174 ;$ HR 2.34, 95\% CI: 1.10-4.98; $P=0.0272$ ). For $O S$, modeling was insufficiently powered, with too few deaths (six of 140, 4.3\%) in the group of patients completing 2 years of venetoclax therapy, hence it was not performed.

Overall, the median duration of venetoclax therapy for patients who discontinued venetoclax early $(n=54)$ was 11.3 months (range, 0.1-24.9; Table 3), compared with 24.4 months (range, 0.1-27.9) for all patients. In patients discontinuing due to $\mathrm{AE}$, median treatment duration was 11.3 months (range, 0.5-23.3), compared with 16.4 months (range, 4.4-24.9) for patients discontinuing for PD $(P=0.063$, Table 3$)$. The majority of discontinuations occurred after completion of the combination therapy (VenR) phase, with 15 patients discontinuing during the combination therapy period (cycles $1-6,0$ to $<6$ months; one patient was determined as discontinued during postcombination/follow-up but is included in the 0 to $<6$ months group in Table 3), nine of these due to AE.

Table 2. MURANO: cause of death for patients who discontinued treatment.

\begin{tabular}{|c|c|c|}
\hline $\begin{array}{l}\text { Cause of venetoclax } \\
\text { discontinuation }\end{array}$ & Cause of death & $\begin{array}{l}\text { Patients who } \\
\text { discontinued } \\
\text { venetoclax } \\
\text { and died }(n=22)\end{array}$ \\
\hline \multirow[t]{12}{*}{ Adverse event } & Acute respiratory failure & $1(4.5 \%)$ \\
\hline & Cardiac failure & $1(4.5 \%)$ \\
\hline & Colorectal cancer & $2(9.1 \%)$ \\
\hline & Respiratory failure & $1(4.5 \%)$ \\
\hline & $\begin{array}{l}\text { Sepsis secondary to transformed acute } \\
\text { myeloid leukemia }\end{array}$ & $1(4.5 \%)$ \\
\hline & Metastatic malignant melanoma & $1(4.5 \%)$ \\
\hline & $\begin{array}{l}\text { Myelodysplastic syndrome } \\
\text { (related to venetoclax) }\end{array}$ & $1(4.5 \%)$ \\
\hline & Myocardial infarction (related to venetoclax) & x) $1(4.5 \%)$ \\
\hline & Pancreatic carcinoma & $1(4.5 \%)$ \\
\hline & Pneumonia & $1(4.5 \%)$ \\
\hline & Status epilepticus & $1(4.5 \%)$ \\
\hline & Sudden death & $1(4.5 \%)$ \\
\hline Progressive disease & $\begin{array}{l}\text { Progressive disease } \\
\text { Pneumonia }\end{array}$ & $\begin{array}{l}3(13.6) \\
1(4.5 \%)\end{array}$ \\
\hline Death & $\begin{array}{l}\text { Pneumonia } \\
\text { Sudden cardiac death }\end{array}$ & $\begin{array}{l}1(4.5 \%) \\
1(4.5 \%)\end{array}$ \\
\hline Withdrawal by patient & Progressive disease & $1(4.5 \%)$ \\
\hline Physician decision & Sepsis (related to venetoclax) & $1(4.5 \%)$ \\
\hline Other & $\begin{array}{l}\text { Not reported (patient had gastric cancer } \\
\text { and was transferred to palliative care) }\end{array}$ & $1(4.5 \%)$ \\
\hline
\end{tabular}

Discontinuation due to AE was more common earlier (34.5\% [ten of 29] between 0 and $<6$ months vs. $20.7 \%$ [six of 29] for $\geq 18$ months), with a visible trend for fewer discontinuations due to $\mathrm{AE}$ as time progressed (Table 3 ).

Overall, $41 \mathrm{AE}$ led to treatment discontinuation in 32 patients (16.5\%; Table 4); however, the primary reason for early venetoclax discontinuation was only determined as AE for 29 patients (primary reasons for the other three patients were physician decision $[n=2]$ and death $[n=1]$ ). Notably, 22 of 29 (75.9\%) patients had a dose reduction or interrupted treatment before prematurely discontinuing venetoclax due to an AE and 18 of 29 (62.1\%) patients discontinued treatment early due to venetoclax related AE (Online Supplementary Table S2). The most common classes of AE (MedDRA preferred terms) leading to discontinuation were blood and lymphatic system disorders $(7.2 \%)$; neoplasms benign, malignant and unspecified $(2.6 \%)$; general disorders and administration site conditions $(2.1 \%)$; and infections and infestations $(2.1 \%)$. The most common individual AE resulting in treatment discontinuation were neutropenia (six of $194 ; 3.1 \%$ ) and thrombocytopenia (five of 194; $2.6 \%$ ).

Objective response rate (ORR: complete response [CR], $\mathrm{CR}$ with incomplete marrow recovery, partial response [PR], and nodular PR) for patients who prematurely discontinued venetoclax due to AE was $79.3 \%$ (23 of 29; Table 4). Best minimal residual disease (MRD) response in peripheral blood (measured by allele-specific oligonucleotide polymerase chain reaction) was undetectable MRD (uMRD; where the threshold for MRD was defined as one tumor cell per $10^{4}$ white cells) for $62.1 \%$ of these patients (18 of 29), while the UMRD rate at the end of combination treatment response visit was $48.3 \%$ (14 of 29; Table 5). Patients who had an uMRD status on or before venetoclax discontinuation and did not have $\mathrm{PD}$ before venetoclax discontinuation $(n=24)$, had a median PFS calculated from cessation of 26.6 (95\% CI: 4.7-32.4) months. There were no robustly significant baseline demographic predictors for discontinuation due to $\mathrm{AE}$; baseline covariates were not significantly associated with discontinuation due to venetoclax related $\mathrm{AE}$, but duration of response to most recent prior therapy was marginally significant $(P=0.0467)$ for treatment discontinuation due to non-related AE (Online Supplementary Table S3).

Table 3. MURANO: discontinuation of venetoclax treatment for all patients, due to adverse events and due to progressive disease.

\begin{tabular}{|c|c|c|c|}
\hline & \multicolumn{3}{|c|}{ Patients who discontinued venetoclax } \\
\hline & $\begin{array}{c}\text { For any reason } \\
(n=54)\end{array}$ & $\begin{array}{l}\text { Due to AE } \\
(n=29)\end{array}$ & $\begin{array}{c}\text { Due to PD } \\
(n=12)\end{array}$ \\
\hline \multicolumn{4}{|l|}{$\begin{array}{l}\text { Duration of Ven treatment, } \\
\text { months }\end{array}$} \\
\hline Mean (SD) & $11.7(8.0)$ & $10.6(7.1)$ & $15.4(7.8)$ \\
\hline Median (range) & $11.3(0.1-24.9)$ & $11.3(0.5-23.3)$ & $16.4(4.4-24.9$ \\
\hline \multicolumn{4}{|l|}{ Ven discontinuation at } \\
\hline 0 to $<6$ months*, $n(\%)$ & $16(29.6)$ & $10(34.5)$ & $1(8.3)$ \\
\hline 6 to <12 months, n (\%) & $13(24.1)$ & $7(24.1)$ & $4(33.3)$ \\
\hline 12 to $<18$ months, $\mathrm{n}(\%)$ & $9(16.7)$ & $6(20.7)$ & $1(8.3)$ \\
\hline 18 to $<24$ months, $\mathrm{n}(\%)$ & $10(18.5)$ & $5(17.2)$ & $3(25.0)$ \\
\hline$\geq 24$ months & $6(11.1)$ & $1(3.4)$ & $3(25.0)$ \\
\hline
\end{tabular}

* One patient was determined as discontinued during post-combination/follow-up, after discontinuing Ven 33 days after rituximab discontinuation (which occurred on cycle 2 day 1); total Ven treatment duration was 98 days (63 days after rituximab was dosed). AE,: adverse event; PD: progressive disease; SD: standard deviation;Ven: venetoclax. 


\section{Treatment interruption}

Of the 194 patients receiving VenR, 137 (70.6\%) required a total of 316 interruptions to venetoclax treatment. Most patients interrupted treatment multiple times, with only 55 of $137(40.1 \%)$ patients interrupting treatment just once. Of the other 82 of 137 patients, $37(27.0 \%)$ interrupted treatment twice, $20(14.6 \%)$ three times, $12(8.8 \%)$ four times and 13 five or more times $(\mathrm{n}=6$ for five interruptions $[4.4 \%], \mathrm{n}=2$ for six interruptions $[1.5 \%], \mathrm{n}=3$ for seven

Table 4. MURANO: adverse events leading to discontinuation, dose interruption or dose reduction of venetoclax treatment.

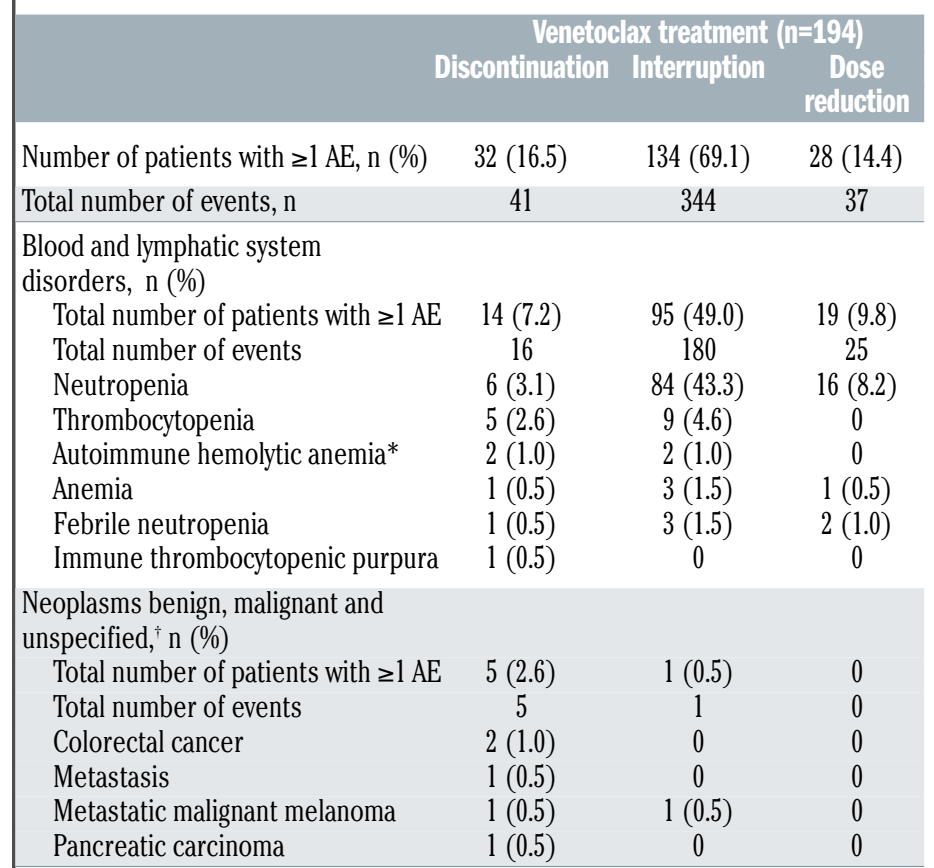

General disorders and administration

site conditions, $\mathrm{n}(\%)$

Total number of events

Asthenia

Pyrexia

Sudden cardiac death*

Sudden death

0

Infections and infestations, $\mathrm{n}(\%)$

Total number of patients with $\geq 1 \mathrm{AE}$

Total number of events

Pneumonia*

Appendicitis

Lung infection

Peritoneal tuberculosis

Upper respiratory tract infection

Gastrointestinal disorders, $\mathrm{n}(\%)$

Total number of patients with $\geq 1 \mathrm{AE} \quad 3(1.5)$

Total number of events

Ascites

Crohn's disease

Diarrhea

Abdominal discomfort

Small intestinal obstruction

$4(2.1)$
4
$1(0.5)$
$1(0.5)$
$1(0.5)$
$1(0.5)$

$\begin{array}{cc}8(4.1) & 0 \\ 8 & 0 \\ 1(0.5) & 0 \\ 3(1.5) & 0 \\ 0 & 0 \\ 0 & 0 \\ & \\ 47(24.2) & 1(0.5) \\ 70 & 1 \\ 8(4.1) & 0 \\ 1(0.5) & 0 \\ 1(0.5) & 0 \\ 0 & 0 \\ 8(4.1) & 1(0.5)\end{array}$

Investigations, $\mathrm{n}(\%)$

Total number of patients with $\geq 1 \mathrm{AE} \quad 2$ (1.0)

Total number of events

Alanine aminotransferase increased

Neutrophil count decreased

Aspartate aminotransferase increased
3
$1(0.5)$

$1(0.5)$

$1(0.5)$

0

0

$16(8.2)$

$4(2.1)$

29

0

0

$9(4.6)$

0
0

0

0

$2(1.0)$

$1(0.5)$

$1(0.5)$

15 (7.7)

25

3 (1.5)

2 (1.0)

$(0.5)$

$1(0.5)$

5 (2.6)

0

$1(0.5)$

$1(0.5)$

$1(0.5)$ interruptions [2.2\%] and $\mathrm{n}=2$ for eight interruptions $[1.5 \%])$. Reason for treatment interruption was not captured specifically in the MURANO study, unless required for management of AE. Most of the interruptions (212 of 316 ) occurred during the combination treatment phase, with 117 of 194 patients (60.3\%) interrupting treatment during combination therapy (212 interruptions) versus 62 of 194 patients $(32.0 \%)$ with interruptions during the monotherapy treatment phase (104 interruptions).

The median consecutive days duration of treatment interruption was 9 days (range, 1-93). Of the 137 patients with treatment interruptions, $61(44.5 \%)$ had interruptions lasting 1-7 consecutive days, $30(21.9 \%)$ had interruptions lasting 8-14 consecutive days, 13 (9.5\%) had interruptions lasting 15-21 consecutive days, 21 (15.3\%) had interruptions lasting 22-28 consecutive days and 12 (8.8\%) had interruptions lasting $>28$ consecutive days (based on the

continued from previous column

Respiratory, thoracic and mediastinal

disorders, $\mathrm{n}(\%)$

Total number of patients with $\geq 1 \mathrm{AE}$

Total number of events

Acute respiratory failure

Hydrothorax

$\begin{array}{ccc}2(1.0) & 2(1.0) & 0 \\ 2 & 2 & 0 \\ 1(0.5) & 0 & 0 \\ 1(0.5) & 0 & 0\end{array}$

Ear and labyrinth disorders, $\mathrm{n}(\%)$

Total number of patients with $\geq 1 \mathrm{AE}$

Total number of events

Vertigo

$1(0.5)$

$1(0.5)$

$\begin{array}{ll}0 & 0 \\ 0 & 0 \\ 0 & 0\end{array}$

Nervous system disorders, n (\%)

Total number of patients with $\geq 1 \mathrm{AE} \quad 1(0.5)$

Total number of events

Status epilepticus

$1(0.5)$
1
$1(0.5)$

$2(1.0)$

2

0

Metabolism and nutrition disorders, $\mathrm{n}(\%)$

Total number of patients with $\geq 1 \mathrm{AE}$

Total number of events

$10(5.2)$

$1(0.5)$

Musculoskeletal and connective tissue disorders, n (\%)

Total number of patients with $\geq 1 \mathrm{AE}$

Total number of events

0
0

11

2

Skin and subcutaneous tissue disorders,

n (\%)

\begin{tabular}{lccc} 
Total number of patients with $\geq 1 \mathrm{AE}$ & 0 & $3(1.5)$ & $1(0.5)$ \\
Total number of events & 0 & 3 & 1 \\
\hline $\begin{array}{l}\text { Cardiac disorders, } \mathrm{n}(\%) \\
\text { Total number of patients with } \geq 1 \mathrm{AE}\end{array}$ & 0 & $2(1.0)$ & 0 \\
$\quad$ Total number of events & 0 & 2 & 0 \\
Hepatobiliary disorders, $\mathrm{n}(\%)$ & & & \\
Total number of patients with $\geq 1 \mathrm{AE}$ & 0 & $2(1.0)$ & $1(0.5)$ \\
Total number of events & 0 & 2 & 1
\end{tabular}

Injury, poisoning and procedural

complications, n (\%)

Total number of patients with $\geq 1 \mathrm{AE}$

Total number of events

Vascular disorders, n (\%)

Total number of patients with $\geq 1 \mathrm{AE}$

Total number of events

$\begin{array}{ccc}0 & 3(1.5) & 0 \\ 0 & 4 & 0\end{array}$

Reproductive system and breast

disorders, $\mathrm{n}(\%)$

Total number of patients with $\geq 1 \mathrm{AE}$

Total number of events

*Includes event for patient whose primary reason for discontinuation was not due to an $\mathrm{AE}$ (primary reason for discontinuation for the patient with autoimmune hemolytic anemia was physician decision, for the patient with sudden cardiac death was death and for the patient with pneumonia was physician decision); $†$ Including cysts and polyps. AE: adverse event. 
longest consecutive number of days per person). Treatment interruption, regardless of duration $(\geq 1, \geq 8, \geq 14$ and $\geq 21$ consecutive days of missed venetoclax treatment) had no statistically significant effect on clinical outcomes (Table 6) compared with no treatment interruption.

Thirty-six (18.6\%) patients who had treatment interruptions later discontinued venetoclax prior to completion of therapy. Previous treatment interruptions did not predict likelihood of prematurely discontinuing venetoclax; discontinuation rate was $26.3 \%$ in patients who previously interrupted treatment versus $31.6 \%$ in patients who did not interrupt treatment (statistically similar; $P=0.4842$ ).

Overall, 134 patients (69.1\%) required treatment interruption for $\mathrm{AE}$ (a total of $344 \mathrm{AE}$ ). The most common types of $\mathrm{AE}$ leading to treatment interruption were blood and lymphatic system disorders $(49.0 \%)$, infections and infestations $(24.2 \%)$, gastrointestinal disorders $(8.2 \%)$ and investigations $(7.7 \%$; Table 4$)$. The most common individual $\mathrm{AE}$ resulting in treatment interruption were neutropenia (84 of 194; 43.3\%), thrombocytopenia (nine of 194; $4.6 \%$ ) and diarrhea (nine of 194; 4.6\%). Notably, 116 of $134(86.6 \%)$ patients interrupted treatment due to venetoclax related AE (Online Supplementary Table S2). Number of prior regimens was the only variable found to be statistically significant $(P=0.0048)$ from the logistic regression model for interruption due to venetoclax related AE (Online Supplementary Table S4). Baseline covariates were not significantly associated with treatment interruption due to AE that were not related to venetoclax (Online Supplementary Table S4). Median time to first onset of AE leading to venetoclax dose interruption was 1.6 months (range, 0.0-22.8).

\section{Dose reduction}

Of the 194 patients receiving VenR, 45 (23.2\%) required dose reductions for venetoclax. A total of 76 dose reductions were required, with some patients having multiple dose reductions (for different reasons); 64 due to $\mathrm{AE}$ (in 40 patients), seven due to unknown reasons (six patients), two due to medication error (two patients), two due to patient choice (one patient) and one due to a medical decision (one patient). Venetoclax dose was reduced to $300 \mathrm{mg}$ for three of $76(3.9 \%)$ dose reductions $(\mathrm{n}=3), 200 \mathrm{mg}$ for 44 of 76 $(57.9 \%)$ dose reductions ( $\mathrm{n}=36), 100 \mathrm{mg}$ for 23 of 76 $(30.3 \%)$ dose reductions $(n=20), 50 \mathrm{mg}$ for two of 76 $(2.6 \%)$ dose reductions $(n=2)$ and $20 \mathrm{mg}$ for four of 76 $(5.3 \%)$ dose reductions $(n=4)$. Forty-five dose reductions (in 30 patients) occurred during the combination treatment phase, compared with 31 dose reductions (in 19 patients) during the monotherapy treatment phase.
Dose reduction had no statistically significant effect on PFS ( $n=165$; HR 1.19, 95\% CI: 0.65-2.18; $P=0.5765$ ) or OS ( $n=192$; HR 0.91, 95\% CI: 0.35-2.38;P=0.8519) compared with patients with no dose reduction. Following the first dose reduction, $26(57.8 \%)$ patients returned to the original dose; median duration from the first dose reduction before returning to the original dose was 16.5 days (range, $1-293)$. Of the 19 patients (42.2\%) who never returned to the original dose, one patient subsequently discontinued venetoclax, eight interrupted venetoclax treatment, three interrupted and then discontinued venetoclax treatment and seven remained on the reduced dose $(200 \mathrm{mg}$ for six patients [ $85.7 \%$ ] and $100 \mathrm{mg}$ for one patient [14.3\%]) until treatment completion.

Although the reason for dose reduction was AE for 40 patients, information on type of $\mathrm{AE}$ and onset of $\mathrm{AE}$ was only available for 28 patients (37 AE). Dose reductions in 25 of $28(89.3 \%)$ patients were due to venetoclax related AE (Online Supplementary Table S2). The most common types of $\mathrm{AE}$ and the most common individual $\mathrm{AE}$ resulting in dose reduction (neutropenia [16 of 194; 8.2\%], febrile neutropenia [two of 194; 1.0\%] and diarrhea [two of 194;

Table 5. MURANO: best overall response and minimal residual disease status (ASO-PCR - peripheral blood) for patients who discontinued venetoclax due to adverse events.

Patients who discontinued
duc to $\mathrm{AE}(\mathrm{n}=29)$

Best overall response (investigator-assessed), n (\%)

Objective response rate

Complete response

$23(79.3)$

Complete response with incomplete

bone marrow recovery

$3(10.3)$

Partial response

$2(6.9)$

Nodular partial response

$17(58.6)$

Stable disease

$1(3.4)$

Progressive disease

$2(6.9)$

$1(3.4)$

Missing

$3(10.3)$

Best MRD response, $\mathrm{n}(\%)$

Undetectable

$18(62.1)$

Positive

$3(10.3)$

Missing

$8(27.6)$

MRD response at end of combination treatment

response visit, $\mathrm{n}(\%)$

Undetectable

$14(48.3)$

Positive

Missing $9(31.0)$

*At the 9-month time point. AE: adverse event; ASO-PCR: allele-specific oligonucleotide polymerase chain reaction; MRD: minimal residual disease.

Table 6. MURANO: impact of interruption of venetoclax treatment versus no interruption on outcomes for all patients.

\begin{tabular}{|c|c|c|c|c|}
\hline & \multicolumn{4}{|c|}{ Duration of treatment interruption (n=194 patients) } \\
\hline & $\geq 1$ days & $\geq 8$ days & $\geq 14$ days & $\geq 21$ days \\
\hline Patients, n & $137(70.6 \%)$ & $76(39.2 \%)$ & $50(25.8 \%)$ & $34(17.5 \%)$ \\
\hline $\begin{array}{l}\text { Progression-free survival } \\
\text { Events, n (\%) } \\
\text { HR }(95 \% \mathrm{CI}) \\
P \text {-value }\end{array}$ & $\begin{array}{c}49(35.8) \\
0.67(0.38-1.19) \\
0.1709\end{array}$ & $\begin{array}{c}29(38.2) \\
1.01(0.59-1.71) \\
0.9741\end{array}$ & $\begin{array}{c}20(40.0) \\
0.92(0.51-1.65) \\
0.7671\end{array}$ & $\begin{array}{c}13(38.2) \\
0.82(0.41-1.65) \\
0.5753\end{array}$ \\
\hline $\begin{array}{l}\text { Overall survival } \\
\text { Events, } \mathrm{n}(\%) \\
\text { HR }(95 \% \mathrm{CI}) \\
P \text {-value }\end{array}$ & $\begin{array}{c}17(12.4) \\
0.97(0.43-2.21) \\
0.9474 \\
\end{array}$ & $\begin{array}{c}11(14.5) \\
1.35(0.60-3.02) \\
0.4646 \\
\end{array}$ & $\begin{array}{c}8(16.0) \\
1.47(0.63-3.45) \\
0.3730 \\
\end{array}$ & $\begin{array}{c}5(14.7) \\
1.31(0.46-3.73) \\
0.6193 \\
\end{array}$ \\
\hline
\end{tabular}

CI: confidence interval; HR: hazard ratio. 
$1.0 \%]$ ) are presented in Table 4 . There were no significant baseline demographic predictors for dose reduction due to venetoclax related AE (Online Supplementary Table S4) and logistic regression modeling was not performed for dose reduction due to $\mathrm{AE}$ that were not related to venetoclax, due to small patient numbers $(n=3)$. Median time to first onset of AE leading to venetoclax dose reduction was 3.99 months (range, 0.3-20.5).

\section{Relative dose intensity}

Median relative dose intensity (total dose received by patients $\div$ expected total target dose, starting from first day of VenR combination therapy until the last day of venetoclax treatment or clinical cut-off, whichever is earlier) was 97.4\% (range, 26.4-100.0; $n=189$ ). Relative dose intensity was classified into four categories; distribution of PFS were similar among these categories (Table 7), indicating that there was no compounded effect of both dose reduction and interruption. This analyses was not performed for OS as modeling was insufficiently powered, with only six deaths among the patients who completed venetoclax treatment.

\section{Cumulative exposure}

Cumulative treatment (number of 28-day cycles) received by patients (excluding those discontinuing for $\mathrm{PD}$ ) was statistically significantly associated with reduced risk of both PFS and OS events (PFS: $n=167, \mathrm{HR} 0.93,95 \% \mathrm{CI}$ : $0.88-0.99, P=0.0263$; OS: $\mathrm{n}=161$, HR $0.85,95 \%$ CI: $0.78-$ $0.92, P<0.0001)$.

\section{Discussion}

The results of these analyses highlight the importance of appropriately managing treatment modifications to ensure optimal outcomes for patients receiving targeted treatment for CLL. Treatment discontinuation prior to completing the MURANO regimen (fixed duration of 2 years of venetoclax) was associated with impaired PFS, while improved PFS and OS were associated with greater cumulative venetoclax treatment exposure. PFS was also statistically significantly lower with discontinuation specifically due to AE. The lack of a significant effect of treatment interruption and dose reduction on outcome highlights the importance of management of AE to allow alternative options such as dose modification or re-initiation of therapy following a treatment break, to maximize the duration of therapy.

Early discontinuation of venetoclax was reported in $28 \%$ of patients participating in the MURANO study, similar to the $35 \%$ discontinuation rate (median followup of 10 months) reported in a real-world study of venetoclax treatment. ${ }^{32}$ It is not clearly indicated whether discontinuation in that study was premature discontinuation (as in the MURANO study) or whether some of these patients were receiving venetoclax monotherapy, where treatment was until progression. The most common reason for discontinuation in the real-world study was PD $(17 \%)$, with $4 \%$ reporting discontinuation due to toxicity. ${ }^{32}$ In contrast, the MURANO data indicate that most patients who discontinue venetoclax prematurely do so due to AE (14.9\%), with PD being the second most common cause of discontinuation $(6.2 \%)$. This emphasizes the difficulty of assessing the impact of treatment discontinuation due to toxicity or PD based on clinical studies and real-world data, especially given that there are currently insufficient real-world data to assess how venetoclax discontinuation due to toxicity affects outcomes. ${ }^{32}$ Furthermore, differences in data reported for the MURANO study compared with real-world data reflects the limitations of cross-comparing data without accounting for differences in patient population and treatment schedule.

In the MURANO study, treatment interruption occurred in 137 of 194 (70.6\%) of patients. Venetoclax treatment interruption (for $\geq 1, \geq 8, \geq 14$ and $\geq 21$ consecutive days) showed no statistically significant effect on clinical outcomes (PFS and OS). The rate of interruptions occurring in MURANO is higher than the rate reported in two real-world studies of venetoclax (41\% [including patients with dose reductions] and $32 \%$, respectively). ${ }^{33,34}$ Two explanations potentially account for this difference: firstly, the more stringent reporting of interruptions within a clinical trial; and secondly, the stringent protocol requirements for mild asymptomatic neutropenia mandated for the MURANO study (see the Online Supplementary Appendix), which might contrast with realworld management, where treatment is typically continued with the addition of granulocyte colony-stimulating factor support. Real-world studies of venetoclax provide further evidence that temporary interruption of treatment (length of interruption not disclosed for one study; median 7 days [range, 1-132] for the other) does not have an impact on PFS. 33,34

Dose reduction occurred in 45 of 194 (23.2\%) of patients in the MURANO study and had no statistically significant effect on PFS or OS. The number of patients requiring dose reductions in MURANO is similar to the $29 \%$ reported in the Roeker et al. real-world study of venetoclax. ${ }^{30}$ Furthermore, the real-world studies of venetoclax support the findings from MURANO that dose

Table 7. MURANO: landmark analysis of progression-free surviva by venetoclax relative dose intensity quartiles.

\begin{tabular}{|c|c|c|c|c|}
\hline & $\begin{array}{c}\operatorname{Min}(26.4 \%)- \\
<01(93.6 \%) \\
n=33\end{array}$ & $\begin{array}{c}\text { Q1 }(93.6 \%)- \\
<\text { Median (98.1\%) } \\
n=35\end{array}$ & $\begin{array}{c}\text { Median }(98.1)- \\
<03(99.5 \%) \\
n=34\end{array}$ & $\begin{array}{c}Q 3(99.5 \%)- \\
\operatorname{Max}(100.0 \%) \\
n=35\end{array}$ \\
\hline Events, n (\%) & $8(24.2)$ & $7(20.0)$ & $9(26.5)$ & $11(31.4)$ \\
\hline Kaplan-Meier median, months $(95 \% \mathrm{CI})$ & NE (22.9-NE) & NE (28.1-NE) & 27.3 (18.8-NE) & 27.7 (22.3-NE) \\
\hline $\mathrm{HR}(95 \% \mathrm{CI})$ & 1.0 & $0.57(0.13-2.49)$ & $1.01(0.20-5.01)$ & $0.95(0.28-3.26)$ \\
\hline$P$-value & 1.0 & 0.4575 & 0.9952 & 0.9331 \\
\hline
\end{tabular}

The landmark analysis was performed to study the effect of relative dose intensity on progression-free survival (PFS).The patients who completed venetoclax treatment and had not progressed or were censored at the last dose of venetoclax, were included. The PFS was calculated from the last dose of venetoclax to the first occurrence of progression or death from any cause. CI: confidence interval; HR: hazard ratio; Max: maximum; Min: minimum; NE: not estimable; PFS: progression-free survival; Q: quartile. 
reduction does not result in inferior PFS (Online Supplementary Table S5). ${ }^{33,34}$

Our literature review revealed evidence that treatment discontinuation of ibrutinib may be associated with poor survival outcomes; the impact of ibrutinib discontinuation may be significantly modified by factors such as the reason for discontinuation and the number of prior therapies received (Online Supplementary Table S1). The impact of ibrutinib interruption is harder to quantify from the literature (Online Supplementary Table S1); the phase III RESONATE study reported an impact on PFS with treatment interruption $\geq 8$ consecutive days ${ }^{16}$ while a phase II trial indicated that outcomes were not compromised by treatment interruption. ${ }^{19}$ Some real-world datasets also suggest an impact of interruption on outcomes, ${ }^{21,23,28}$ while others do not. ${ }^{24,26}$ Similarly the impact of ibrutinib dose reduction is difficult to quantify from the literature (Online Supplementary Table S5); one interventional and one real-world study suggest that there is no survival detriment with dose reduction, ${ }^{19,23}$ whereas inferior PFS is indicated in two real-world studies..$^{24,28}$

The current literature consists of a mixture of clinical trials and real-world data; therefore patient characteristics, management of $\mathrm{AE}$ and the timing of data collection/availability of data may vary significantly. This could explain the inconsistencies in the data, where numerous studies have reported conflicting results, making conclusions particularly difficult to draw. In addition to this, it is important to also note the variation in the definitions used for dose reduction. While some studies indicate that data are representative of early dose reductions (e.g., within the first 8 weeks of initiating therapy ${ }^{28}$ ), other studies, such as MURANO, look at dose reductions occurring throughout therapy. Furthermore the lack of information indicating the permanence of dose reductions, or whether all dose reductions experienced by a patient were taken into account or not, could provide some explanation for inconsistencies in the data reported.

Literature assessing the impact of discontinuation and interruption on outcomes was limited for both idelalisib and duvelisib. Discontinuation data from clinical trials of idelalisib showed similar OS in one study and reduced PFS in another, ${ }^{35,36}$ while real-world data indicated an effect on survival dependent upon reason for treatment discontinuation. ${ }^{37}$ Phase III data suggest an association between idelalisib interruption and longer PFS and OS (with length of interruption potentially having an impact on outcome), but this is difficult to confirm with only one study available (Online Supplementary Table S1). ${ }^{38}$ Similarly, conclusions are hard to draw for duvelisib treatment interruptions with only one study reporting the impact on PFS (which is similar regardless of duration of interruption [>1 week vs. >2 weeks]; Online Supplementary Table S1). ${ }^{39}$

The analyses presented here are the first to be conducted from MURANO for the impact of venetoclax dose modification or premature discontinuation. Since analyses were performed retrospectively, without type-I error control, the results should be interpreted with caution. This manuscript also provides the first literature review on this topic to collate the numerous articles and provide context for the MURANO analyses. Limited published data for the impact of venetoclax discontinuation and interruption on survival hindered comparison of the MURANO results with other studies of venetoclax. Moreover, the review was further limited by the inability to perform a meta-analysis in order to compare the MURANO results with studies of other targeted therapies (ibrutinib, idelalisib and duvelisib).

In conclusion, these analyses provide the first comprehensive account of the impact of discontinuation, treatment interruption and dose reduction of oral targeted treatment on PFS and/or OS in patients with $1 \mathrm{~L}$ or $\mathrm{R} / \mathrm{R}$ CLL. These data highlight the importance of prompt treatment modification upon development of $\mathrm{AE}$ with venetoclax, as effective management of $\mathrm{AE}$ with treatment interruption or dose reduction allows patients to continue venetoclax treatment and avoid the negative impact of treatment discontinuations on outcomes.

\section{Disclosure}

ARM consults for or advises for AbbVie, AstraZeneca, Celgene, Genentech, Janssen, Loxo, PCYC, Sunesis and TG therapeutics, and has received research funding from AbbVie, AstraZeneca, Celgene, DTRM, Genentech, Janssen, Loxo, $P C Y C$, Sunesis and TG Therapeutics; JPS consults for or advises for AbbVie, Acerta, AstraZeneca, Bayer Health, Celgene, F. Hoffmann-La Roche, Pharmacyclics and TG Therapeutics, has received research funding from AbbVie, Acerta, AstraZeneca, Celgene, Pharmacyclics and TG Therapeutics, has received travel support from AbbVie, AstraZeneca and Celgene, and has role or activity within Pfizer; JMLB, $M W$ and YM are employees and stockholders of Genentech; SYK is an employee and stockholder of AbbVie, and has received travel support from AbbVie; $K H$ and $M B$ are employees and stockholders of $F$. Hoffmann-La Roche; QZ is an employee and stockholder of Edwards Life Sciences, and consults or advises for Genentech, JFS has received honoraria from and consults/advises for AbbVie, BMS, Celgene, F. Hoffmann-La Roche, Gilead, Janssen, Mei Pharma, Sunesis and Takeda, has participated in speaker's bureaus for AbbVie and F. Hoffmann-La Roche, has received research funding from AbbVie, Celgene, F. HoffmannLa Roche and Janssen, has provided expert testimony for $F$. Hoffmann-La Roche, and has received travel support from AbbVie, BMS, F. Hoffmann-La Roche and Janssen.

\section{Contributions}

All authors were involved in the analysis and interpretation of the data, as well as review and/or revision of the manuscript.

\section{Acknowledgments}

The authors would like to thank the patients and their families, investigators, study coordinators and support staff, and MURANO study team members.

\section{Funding}

Venetoclax is being developed in collaboration between Genentech and AbbVie. Genentech and AbbVie provided financial support for the study and participated in the design, study conduct, analysis, and interpretation of data, as well as the writing, review and approval of the manuscript. Medical writing support was provided by Rachel Dobb of Ashfield MedComms, an Ashfield Health company and was funded by F. HoffmannLa Roche Ltd. 


\section{References}

1. Eichhorst B, Robak T, Montserrat E, et al. Chronic lymphocytic leukaemia: ESMO Clinical Practice Guidelines for diagnosis, treatment and follow-up. Ann Oncol. 2015;26 (Suppl 5):v78-84.

2. Siegel RL, Miller KD, Jemal A. Cancer statistics, 2017. CA Cancer J Clin. 2017;67(1):730.

3. Dreger P. Allotransplantation for chronic lymphocytic leukemia. Hematology Am Soc Hematol Educ Program. 2009:602-609.

4. National Comprehensive Cancer Network Clinical Practice Guidelines in Oncology (NCCN Guidelines): Chronic Lymphocytic Leukemia/Small Lymphocytic Lymphoma. Version 2.2019. NCCN website. www.nccn.org/professionals/physician_gls/ pdf/cll.pdf. Published October, 2018. Accessed August, 2019.

5. Souers AJ, Leverson JD, Boghaert ER, et al. ABT-199, a potent and selective BCL-2 inhibitor, achieves antitumor activity while sparing platelets. Nat Med. 2013;19(2):202208.

6. Brown JR. Ibrutinib (PCI-32765), the first BTK (Bruton's tyrosine kinase) inhibitor in clinical trials. Curr Hematol Malig Rep. 2013;8(1):1-6.

7. Liu P, Cheng H, Roberts TM, et al. Targeting the phosphoinositide 3-kinase pathway in cancer. Nat Rev Drug Discov. 2009;8(8):627644.

8. Montero J, Letai A. Why do BCL-2 inhibitors work and where should we use them in the clinic? Cell Death Differ. 2018;25(1):56-64

9. Fischer K, Al-Sawaf O, Bahlo J, et al. Venetoclax and obinutuzumab in patients with CLL and coexisting conditions. N Engl J Med. 2019;380(23):2225-2236.

10. Stilgenbauer S, Eichhorst B, Schetelig J, et al. Venetoclax in relapsed or refractory chronic lymphocytic leukaemia with $17 p$ deletion: a multicentre, open-label, phase 2 study. Lancet Oncol. 2016:17(6):768-778.

11. Stilgenbauer S, Eichhorst B, Schetelig J, et al. Venetoclax for patients with chronic lymphocytic leukemia with $17 p$ deletion: results from the full population of a phase II pivotal trial. J Clin Oncol. 2018;36(19):1973-1980.

12. Roberts AW, Seymour JF, Eichhorst B, et al. Pooled multi-trial analysis of venetoclax efficacy in patients with relapsed or refractory chronic lymphocytic leukemia. Blood. 2016;128(22):3230

13. Jones J, Choi MY, Mato AR, et al. Venetoclax (VEN) Monotherapy for patients with chronic lymphocytic leukemia (CLL) who relapsed after or were refractory to Ibrutinib or Idelalisib. Blood. 2016:128(22):637.

14. Jain P, Keating M, Wierda W, et al. Outcomes of patients with chronic lymphocytic leukemia after discontinuing ibrutinib. Blood. 2015;125(13):2062-2067.

15. Maddocks KJ, Ruppert AS, Lozanski G, et al.
Etiology of ibrutinib therapy discontinuation and outcomes in patients With chronic lymphocytic leukemia. JAMA Oncol. 2015;1(1):80-87.

16. Barr PM, Brown JR, Hillmen P, et al. Impact of ibrutinib dose adherence on therapeutic efficacy in patients with previously treated CLL/SLL. Blood. 2017;129(19):2612-2615.

17. Jain P, Thompson PA, Keating M, et al. Longterm outcomes for patients with chronic lymphocytic leukemia who discontinue ibrutinib. Cancer. 2017;123(12):2268-2273.

18. O'Brien SM, Byrd JC, Hillmen P, et al. Outcomes with ibrutinib by line of therapy and post-ibrutinib discontinuation in patients with chronic lymphocytic leukemia: phase 3 analysis. Am J Hematol. 2019;94(5):554-562

19. Ahn IE, Basumallik N, Tian X, et al. Clinically-indicated ibrutinib dose interruptions and reductions do not compromise long-term outcomes in CLL. Blood. 2019;133(22):2452-2455.

20. Sandoval-Sus JD, Chavez JC, Dalia S, et al. Outcomes of patients with relapsed/refractory chronic lymphocytic leukemia after ibrutinib discontinuation outside clinical trials: a single institution experience. Blood. 2015;126(23):2945

21. Thompson PA, Levy V, Tam CS, et al. The impact of atrial fibrillation on subsequent survival of patients receiving ibrutinib as treatment of chronic lymphocytic leukemia (CLL): An international study. Blood. 2016; 128(22):3242

22. Akhtar OS, Torka P, Bhat SA, et al. Disease progression on ibrutinib therapy is associated with a poor clinical outcome in chronic lymphocytic leukemia (CLL) patients managed in standard clinical practice. Blood. 2017;130(Suppl 1):S5350

23. Follows GA, CLL Forum UK. Ibrutinib for relapsed/refractory CLL: an update of the UK and Ireland analysis of outcomes in 315 patients. Haematologica. 2017;35(S2):238239.

24. Rhodes J, Barr PM, Ujjani CS, et al. The impact of front-line ibrutinib dose reduction and interruption on outcomes in chronic lymphocytic leukemia (CLL) patients. Blood. 2017;130(Suppl 1):S4313.

25. Sharman JP, Black-Shinn JL, Clark J, et al. Understanding ibrutinib treatment discontinuation patterns for chronic lymphocytic leukemia. Blood. 2017;130(Suppl 1):S4060.

26. Winqvist M, Andersson P-O, Asklid A, et al. Real-world results on ibrutinib in relapsed/refractory CLL: 30-month followup of 95 Swedish patients treated in a compassionate use program. HemaSphere. 2018;2(Suppl 1):S128-129.

27. Hampel PJ, Ding W, Call TG, et al. Rapid disease progression following discontinuation of ibrutinib in patients with chronic lymphocytic leukemia treated in routine clinical practice. Leuk Lymphoma. 2019;60: (11):2712-2719.
28. Williams AM, Baran AM, Casulo C, et al Ibrutinib dose adherence and therapeutic efficacy in non-Hodgkin lymphoma: a single-center experience. Clin Lymphoma Myeloma Leuk. 2019;19(1):41-47.

29. Seymour JF, Kipps TJ, Eichhorst B, et al. Venetoclax-rituximab in relapsed or refractory chronic lymphocytic leukemia. N Engl J Med. 2018;378(12):1107-1120.

30. Seymour JF, Kipps TJ, Eichhorst BF, et al. Four-year analysis of Murano study confirms sustained benefit of time-limited venetoclax-rituximab (VenR) in relapsed/refractory (R/R) chronic lymphocytic leukemia (CLL). Blood. 2019;134(Suppl 1):S355.

31. Lew TE, Anderson MA, Lin VS, et al. Undetectable peripheral blood MRD should be the goal of venetoclax in CLL, but attain ment plateaus after 24 months. Blood Adv. 2020;4(1):165-173.

32. Mato AR, Tam CS, Allan JN, et al. Disease and patient characteristics, patterns of care, toxicities, and outcomes of chronic lymphocytic leukemia (CLL) patients treated with venetoclax: A multicenter study of 204 patients. Blood Adv. 2017;130(Suppl 1):S4315.

33. Eyre TA, Kirkwood AA, Gohill S, et al. Efficacy of venetoclax monotherapy in patients with relapsed chronic lymphocytic leukaemia in the post-BCR inhibitor setting: a UK wide analysis. Br J Haematol. 2019;185(4):656-669.

34. Roeker LE, Fox CP, Eyre TA, et al. Tumor lysis, adverse events, and dose adjustments in 297 venetoclax-treated CLL patients in routine clinical practice. Clin Cancer Res. 2019;25(14):4264-4270.

35. Brown JR, Ghia P, Jones JA, et al. Outcomes of patients with relapsed and refractory chronic lymphocytic leukemia (CLL) who discontinue idelalisib treatment. J Clin Oncol. 2016;34(Suppl):S7531.

36. Thompson PA, Stingo F, Keating MJ, et al. Outcomes of patients with chronic lymphocytic leukemia treated with first-line idelalisib plus rituximab after cessation of treatment for toxicity. Cancer. 2016;122(16): 2505-2511.

37. Bange E, Nabhan C, Brander DM, et al. Realworld evidence for durable treatment responses after toxicity related discontinuation of idelalisib. Blood. 2017;130(Suppl 1):S4325.

38. Ma S, Chan RJ, Ye W, et al. Survival outcomes following idelalisib interruption in the treatment of relapsed or refractory indolent non-Hodgkin's lymphoma and chronic lymphocytic leukemia. Blood. 2018;132 (Suppl 1):S3149.

39. Flinn I, Montillo M, Illés Á, et al. Effect of dose modifications on response to duvelisib in patients with relapsed/refractory $(\mathrm{R} / \mathrm{R})$ CLL/SLL in the DUO trial. J Clin Oncol. 2019;37(Suppl 15):S7523. 